\title{
Politische Kommunikation im Internet, Web2.0 im Wahlkampf und ePartizipation. Das Politcamp09 am 2./3. Mai 2009 in Berlin
}

Am 2. und 3. Mai 2009 fand in Berlin das erste so genannte Politcamp mit mehr als 700 Teilnehmern statt. Die Konferenz befasste sich mit dem Potenzial internetbasierter Kommunikationsformen für die Politik, insbesondere für den Wahlkampf. Es gab nicht nur anwenderorientierte Tipps, sondern es wurden auch demokratietheoretische Fragestellungen diskutiert. Im Superwahljahr 2009 ist das Interesse um den „Wahlkampf 2.0“ von USPräsident Barack Obama und die möglichen Erkenntnisse für den deutschen Wahlkampf ungebrochen. Es ist deshalb nicht erstaunlich, dass Wahlkämpfer hierzulande bestrebt sind zu erfahren, wie sie mit den „neuen“ Instrumenten des Internets ihre Anhängerschaft mobilisieren, neue Unterstützer gewinnen und Wahlen für sich entscheiden können.

Organisiert wurde das Politcamp von einer überparteilichen Gruppe von Politikbegeisterten, finanziert durch Teilnehmergebühren und Sponsoren. Die Veranstaltung war in unterschiedliche thematische Panels unterteilt, die von den Teilnehmern selbst gestaltet wurden (das zugrunde liegende Konzept wird auch als „OpenSpace oder „BarCamp“ bezeichnet).

Die zwei Konferenztage wurden jeweils mit einem besonderen Highlight eröffnet. Am ersten Tag fand ein „Twitter-Panel“ statt. Twitter bezeichnet ein so genanntes Microblogging-Tool, mit dem SMS-Nachrichten an eine große Menge von Lesern verteilt werden können. Auf dem Twitter-Panel diskutierten mit Volker Beck (MdB, Grüne), Matthias Groote (MdEP, SPD) und Frank Schäffler (MdB, FDP) drei prominente Politik-Twitterer über die Wirkung dieses neuen Mediums im Wahlkampf. Beck sah in Twitter eine einfache wie sinnvolle Möglichkeit für Politiker, direkt mit Wählern zu kommunizieren, denn kaum ein anderes Medium erlaube so schnelle und unkomplizierte Kommunikation: „Nicht für alles lohnt es sich, eine Pressemitteilung zu schreiben, und mit Twitter hat man sofort Feedback von den Bürgern." Nach Ansicht von Groote sei eine neue Kultur der ePartizipation, also des Dialogs und der Teilhabe der Bürger an der Politik durch elektronische Medien notwendig. Und diese beschränke sich eben nicht nur auf den Wahlkampf.

Das Thema „Demokratie im Internet“ wurde im Rahmen der Diskussionen des Politcamps in vielen unterschiedlichen Facetten behandelt. Am Nachmittag des ersten Tages fand beispielsweise ein Panel mit dem Titel „Politikerbashing im Web 2.0“ statt. Motivation für die Ideengeberin des Panels, die Kommunalpolitikerin Angelika Dorsch, war ihr Eindruck, dass die in der Web 2.0-Community oft gepflegten Vorurteile und Ressentiments gegenüber Politik, Politikern und Parteien Politikverdrossenheit forcieren würden. Heftig diskutiert wurde auch die Frage, ob Politiker das Web 2.0 lediglich zur Verbreitung ihrer Parteiwerbung nutzen, oder vielmehr ernsthaft bereit sind, Anregungen aus der Netz-Community in ihre politische Arbeit einzubringen. Unter den Teilnehmern überwog die Einschätzung, dass die Politik viel von den offenen (demokratischen) Strukturen des Internets lernen könnte - zum Beispiel wenn es darum ginge, Parteiprogramme oder Gesetzesinitiativen für eine Diskussion im Netz zu öffnen. Grundsätzlich wurde es aber als positiv eingeschätzt, dass sich viele Politiker durch den Wahlkampf mit den Diskussionsformen im Netz intensiv auseinandersetzen und dies auch die Bereitschaft erhöhen würde, die neuen Dialogformen später in den Parlamenten stärker zu nutzen.

„Die Zukunft (oder das Ende?) des Parteienstaats“ stand im Mittelpunkt eines weiteren Panels. Zur Kritik, die die Teilnehmer am parlamentarischen System der Bundesrepublik 
vorbrachten, zählten unter anderem die herausgehobene Stellung der Parteien bei der Auswahl von Kandidaten und Einschränkungen der Abgeordneten durch die Fraktionsdisziplin. In teils heftigen, aber auch unterhaltsamen Debatten wurde von den zahlreich anwesenden politisch Engagierten klar gemacht, wie wichtig innerparteiliche Demokratie einerseits und Fraktionszusammenhalt andererseits für das Funktionieren von Parteien sind. Politik ist in erster Linie eine Kunst des Kompromissfindens, und Parteien sind dafür ein wichtiger Ort und zudem notwendig, um gefundene Kompromisse durchzusetzen.

Der Sonntag wurde mit einer Elefanten-Runde eröffnet, in der die Verantwortlichen der Parteien für den Internet-Wahlkampf zusammentrafen. Unter den Diskutanten waren Oliver Röseler (Leiter Marketing, CDU). Kajo Wasserhövel (Bundesgeschäftsführer, SPD), Robert Heinrich (Online-Wahlkampfleiter, Grüne), Thomas Scheffler (in Vertretung für den Bundesgeschäftsführer der FDP, Hans-Jürgen Beerfeltz) und Halina Wawzyniak (stellvertretende Parteivorsitzende, Die Linke). Hier wurde die Bedeutung von sozialen Netzwerken wie Facebook oder StudiVZ für den Wahlkampf diskutiert. Bei diesen können Internetnutzer sich Profile anlegen und untereinander verknüpfen. Die Plattformen ergänzen mittlerweile traditionelle Kommunikationsformen im Internet wie E-Mail, da die Internetnutzer direkt über die Aktivitäten von anderen Nutzern informiert werden. Unter den Parteivertretern herrschte Einigkeit darüber, dass durch die sozialen Netzwerke die Hemmschwelle für die Bürger, mit Politikern in Kontakt zu treten, deutlich verringert wird. So führe das Internet dazu, den Dialog mit den Wählern zu vereinfachen. Aufgrund zeitlicher Kapazitäten ist es allerdings für die meisten Politiker kaum möglich, kontinuierlich in (allen) Netzwerken präsent zu sein. Daher werden oft Mitarbeiter beauftragt, die Politikerprofile zu pflegen. Mit Blick auf die Authentizität der politischen Kommunikation sei es dann wichtig, so die Meinung der Zuhörer, dass bei Aktivitäten eindeutig gekennzeichnet werde, „aus wessen Feder" diese stammten.

In den Diskussionen kamen auch aktuelle politische Themen zur Sprache. So kritisierten die Teilnehmer deutlich die von der Bundesregierung geplanten Internet-Sperren für kinderpornographische Seiten. Wasserhövel nahm die Kritik auf und bot an, ausführlich darüber zu diskutieren. In einem gemeinsamen Panel von Alexander Kurz (CDU) und Oliver Zeisberger (SPD) wurde dargelegt, wie in dem kurzen Wahlkampf zur Landtagswahl in Hessen der Online-Wahlkampf die Sicht der Medien auf die Kandidaten verändert hat. Übereinstimmend wurde festgestellt, dass der Wahlkampf im Internet zwar nicht wahlentscheidend, jedoch zum Beispiel dafür wichtig war, den in der Bevölkerung unbekannten SPD-Kandidaten Thorsten Schäfer-Gümbel vorzustellen.

Die Veranstaltung hat gezeigt, dass es möglich ist, über Partei- und Interessengrenzen hinweg über (Netz-)Politik und Beteiligung, die Veränderung demokratischer Prozesse durch das Internet und die Meinungsbildung im und durch das Internet zu diskutieren. Dabei war festzustellen, dass die Herausforderungen für die Politiker aller Parteien ähnlich sind: Im Prinzip geht es einerseits darum, die (technologischen) Methoden des Web 2.0 so zu nutzen, dass aus direkter Kommunikation ein „Mehr“ an Einflussmöglichkeiten für die Bürger entsteht, ohne sich aber im virtuellen Dschungel zu verlieren. Andererseits sollten Politiker das Internet nicht nur als virtuellen Infostand verstehen, sondern als Dialogmedium. 